ISSN 0258-7122

Bangladesh J. Agril. Res. 37(1): 39-48, March 2012

\title{
HETEROSIS AND QUALITATIVE ATTRIBUTES IN WINTER TOMATO (Solanum lycopersicum L.) HYBRIDS
}

\author{
M. R. ISLAM ${ }^{1}$, S. AHMAD ${ }^{2}$ AND M. M. RAHMAN ${ }^{3}$
}

\begin{abstract}
An investigation was carried out at the Research Farm of Olericulture Division of Horticulture Research Centre of Bangladesh Agricultural Research Institute (BARI) to evaluate the heterotic performance in $\mathrm{F}_{1}$ generation of tomato. The hybrids showed significant variation in heterosis. The highest heterobeltiotic effects were observed in the cross $\mathrm{P}_{3} \times \mathrm{P}_{8}(-18.46 \%)$ for earliness, $\mathrm{P}_{1} \times \mathrm{P}_{6}(8.57$ \%) for flowers per cluster, $\mathrm{P}_{2} \times \mathrm{P}_{6}(21.73 \%)$ for fruits per cluster, $\mathrm{P}_{6} \times \mathrm{P}_{7}$ (75.54\%) for plant height, $\mathrm{P}_{5} \times \mathrm{P}_{6}(67.44 \%)$ for fruits per plant, $\mathrm{P}_{9} \times \mathrm{P}_{10}(54.82$ \%) for yield per plant, $\mathrm{P}_{2} \times \mathrm{P}_{8}(21.21 \%)$ for individual fruit weight, $\mathrm{P}_{7} \times \mathrm{P}_{8}$ (3.09 \%) for fruit length, $\mathrm{P}_{3} \times \mathrm{P}_{8}(14.11 \%)$ for fruit diameter and $\mathrm{P}_{1} \times \mathrm{P}_{6}(13.11 \%)$ for brix content. In respect of fruit external characters like shape, pedicel area, shape of pistil scar, blossom end shape genotypes were found diverse. Internal qualitative character like firmness, fleshiness and less seeded and locule numbers were highly variable among the genotypes. Considering all the characters the crosses $\mathrm{P}_{1} \times \mathrm{P}_{8}, \mathrm{P}_{2} \times \mathrm{P}_{6}, \mathrm{P}_{2} \times \mathrm{P}_{7}, \mathrm{P}_{2} \times \mathrm{P}_{8}, \mathrm{P}_{3} \times \mathrm{P}_{8}$ and $\mathrm{P}_{5} \times \mathrm{P}_{6}$ were found suitable for further studies to variety selection.
\end{abstract}

Keywords: Heterosis, qualitative attributes, winter tomato.

\section{Introduction}

Tomato (Solanum lycopersicum L.), one of the most popular and an important vegetable of Bangladesh, is widely grown in many parts of the world because of its taste, high nutritional value, multipurpose uses and commercial importance. Bangladesh produces 137 thousand tons of tomato per year from 15.39 thousand hectares of land with an average yield of $8.90 \mathrm{t} / \mathrm{ha}$ (BBS, 2007). The yield is very low compared to that of other tomato growing countries. For this reason, this crop has received high attention to the researchers for its improvement.

Estimation of heterosis is an important way to assess the performances of the hybrids compared to their parents and is essential to develop high yielding varieties of different crop plants. The development of hybrid varieties with desired characters has proven to be an effective strategy to increase tomato production in the world. In tomato, heterosis has been exploited in $F_{1}$ hybrids to a great extent for more than 50 years in many developed countries like USA, Europe, and Japan. In India, heterosis in tomato resulted in an increased yield of

${ }^{1 \& 2}$ Olericulture Division, Horticulture Research Centre, Bangladesh Agricultural Research Institute (BARI), Gazipur-1701, ${ }^{3}$ Professor, Department of Horticulture, Bangabandhu Sheikh Mujibur Rahman Agricultural University (BSMRAU), Gazipur, Bangladesh.. 
20-50\% (Choudhury and Khanna, 1972). Apart from high total yield $\mathrm{F}_{1}$ hybrids have specific advantages on early yield, number of fruits, size of fruit, improved quality, uniformity, adaptation to adverse climatic condition, resistance to diseases and pests (Tesi et al., 1970).

Keeping this view in consideration, the present study has been undertaken with the following objectives:

1. to determine the heterosis or hybrid vigor of the crossed materials;

2. to select winter hybrid varieties having desired qualities.

\section{Materials and Method}

The experiment was conducted in the research farm of Olericulture Division, Horticulture Research Centre (HRC) of Bangladesh Agricultural Research Institute (BARI), Joydebpur, Gazipur during the winter season (October to March) of 2007-2008. The location of the site was $24.00^{\circ} \mathrm{N}$ Latitude and $90.25^{\circ} \mathrm{E}$ Longitude at an elevation of 8.4 meters from the sea level (Anon., 1995). The climate of the experimental site is suited in the sub-tropical climate zone and characterized by heavy rainfall during the months of May to August and medium to low during the rest of the year. The monthly average minimum and maximum temperatures during the crop period were $17.56^{\circ} \mathrm{C}$ and $28.18^{\circ} \mathrm{C}$, respectively. The monthly average relative humidity was $54.88 \%$, rainfall was $10.59 \mathrm{~mm}$. The soil of the experimental field was clay loam in texture having a $\mathrm{pH}$ around 6.0. Seeds of the selected 19 cross combinations from a half diallel cross without reciprocals and their 14 parental lines were used for the study. Recommended dose of fertilizer (550, 450, and $250 \mathrm{~kg} / \mathrm{ha}$ of urea, TSP, MP, and $10 \mathrm{t} / \mathrm{ha}$ of cowdung) were applied (Razzaque et al., 2000).

A tri-replicated experiment was laid out in the Randomized Complete Block Design. The unit plot size was $4.8 \mathrm{~m}$. x 1m accommodating 20 plants in a plot having row to row and plant to plant spacing of 60 and $40 \mathrm{~cm}$, respectively. The unit plots and blocks were separated by $50 \mathrm{~cm}$ and $1 \mathrm{~m}$ space, respectively. Treatments were randomly allotted in each block. Thirty days old seedlings were transplanted in the experimental field on 10 November 2007. Harvesting was started from 77 days after transplanting (27 January 2008) and continued for 35 days. Five plants were randomly selected from each unit plot for recording data.

For estimation of heterosis in each character, the mean values of $19 \mathrm{~F}_{1} \mathrm{~s}$ were compared with better parent (BP) for heterobeltiosis. MSTAT-C program was used for statistical analysis of the data. The recorded data for different characters were subjected to variance analysis. Genotypes means were compared by Tukey's Honestly Significant Difference Test and Coefficient (CV \%) were also estimated as suggested by Gomez and Gomez (1984). 


\section{Results and Discussion}

\section{A. Heterosis \\ Days to 50 \% flowering}

All 19 combinations showed negative heterobeltiosis of which 18 are highly significant for this trait (Table 1). The highest negative heterosis was observed in the combination $\mathrm{P}_{3} \times \mathrm{P}_{8}(-18.46 \%)$ and the lowest was in $\mathrm{P}_{2} \times \mathrm{P}_{6}(-3.76 \%)$. Ahmad (2002) reported negative heterosis for days to $50 \%$ flowering.

\section{Days to last harvest}

Six cross combinations out of 19 showed significant negative heterobeltiosis for this trait. Seventeen showed negative, one showed positive and another one showed no heterosis (Table 1). Heterosis for this trait ranged from - 13.95 to 4.9 percent. The highest negative heterosis was observed in the combination $\mathrm{P}_{6} \times \mathrm{P}_{9}$ $(-13.95 \%)$. The highest positive heterotic effect was observed in the combination $\mathrm{P}_{4} \times \mathrm{P}_{6}(4.9 \%)$.

\section{Plant height (cm)}

In the case of plant height, 17 cross combinations out of 19 showed positive heterosis over better parent, but only 10 were significantly different. The heterotic effects ranged from -7.51 to 75.54 percent (Table 1). The highest significant positive heterosis was observed in $\mathrm{P}_{6} \times \mathrm{P}_{7}(75.54 \%)$ followed by $\mathrm{P}_{2} \mathrm{x}$ $\mathrm{P}_{7}$ (40.26\%). The highest significant negative heterosis for plant height at last harvest was observed in $\mathrm{P}_{7} \times \mathrm{P}_{9}$. Ahmad (2002) recorded appreciable heterosis for plant height.

\section{Flowers per cluster}

Four cross combinations out of 19 showed significant positive better parent heterosis for flowers per cluster (Table 1). The heterosis over better parent ranged from -13.22 to 8.57 percent. The highest positive heterosis was observed in $\mathrm{P}_{1} \times \mathrm{P}_{6}(8.57 \%)$ followed by $\mathrm{P}_{6} \times \mathrm{P}_{9}(6.93 \%)$. The highest significant negative heterosis was observed in $\mathrm{P}_{7} \times \mathrm{P}_{9}(-13.57 \%)$. These results also support the findings of Ahmad (2002).

\section{Fruits per cluster}

Eleven cross combinations out of 19 showed significant positive better parent heterosis for fruits per cluster (Table 1). The heterosis over better parent ranged from -27.93 to 21.73 percent. The highest positive heterosis was observed in $\mathrm{P}_{3} \mathrm{X}$ $\mathrm{P}_{6}(21.73 \%)$ followed by $\mathrm{P}_{7} \times \mathrm{P}_{8}(17.94 \%)$. The highest significant negative heterosis was observed in $\mathrm{P}_{11} \times \mathrm{P}_{12}$ (-27.93\%). Resende et al. (2000) also found appreciable heterosis for fruits per cluster in tomato. 


\section{Fruits per plant}

Eleven cross combinations out of 19 showed significant positive heterosis for fruits per plant (Table 2). Range of better parent heterosis was -43.30 to 67.44 percent. The highest significant positive heterosis was observed in $\mathrm{P}_{5} \mathrm{XP}_{6}$ (67.44\%) followed by $\mathrm{P}_{1} \times \mathrm{P}_{8}$ (49.02\%). The highest significant negative better parent heterosis was observed in $\mathrm{P}_{7} \times \mathrm{P}_{9}(-43.30 \%)$. Heterosis for fruits per plant in tomato was also reported by Ahmad (2002).

Table 1. Percent heterosis over better parent in 19 tomato hybrids for morphological characters.

\begin{tabular}{|c|c|c|c|c|c|}
\hline Crosses & $\begin{array}{c}\text { Days to } \\
50 \% \\
\text { flowering }\end{array}$ & $\begin{array}{c}\text { Days to } \\
\text { last } \\
\text { harvest }\end{array}$ & $\begin{array}{l}\text { Plant height } \\
\text { (cm) }\end{array}$ & $\begin{array}{c}\text { No. of } \\
\text { flowers/cluster }\end{array}$ & $\begin{array}{l}\text { No. of } \\
\text { fruits/cluster }\end{array}$ \\
\hline $\mathrm{P}_{1} \mathrm{X} \mathrm{P}_{6}$ & $-9.14 * *$ & $-11.36 * *$ & 12.46 & $8.57 * *$ & $10.01 * *$ \\
\hline $\mathrm{P}_{1} \mathrm{X} \mathrm{P}_{7}$ & $-9.84 * *$ & -3.81 & 16.74 & $-7.43 * *$ & $2.51 * *$ \\
\hline $\mathrm{P}_{1} \mathrm{X} \mathrm{P}_{8}$ & $-7.69 * *$ & -7.79 & $17.90 *$ & $-1.76 * *$ & $15.00 * *$ \\
\hline $\mathrm{P}_{2} \mathrm{X} \mathrm{P}_{6}$ & $-3.76 *$ & -6.20 & $29.24 * *$ & $-0.957 *$ & $-5.56 * *$ \\
\hline $\mathrm{P}_{2} \mathrm{X} \mathrm{P}_{7}$ & $-9.84 * *$ & -3.81 & $40.26 * *$ & $-6.186 * *$ & $-10.25 * *$ \\
\hline $\mathrm{P}_{2} \mathrm{X} \mathrm{P}_{8}$ & $-15.72 * *$ & -7.79 & $28.15 * *$ & $-11.49 * *$ & $8.10 * *$ \\
\hline $\mathrm{P}_{3} \mathrm{X} \mathrm{P}_{6}$ & $-12.36 * *$ & $-11.36 * *$ & 15.38 & $-1.79 * *$ & $21.73 * *$ \\
\hline $\mathrm{P}_{3} \mathrm{X} \mathrm{P}_{8}$ & $-18.46 * *$ & $-10.48 * *$ & $21.57 *$ & -0.49 & $8.11 * *$ \\
\hline $\mathrm{P}_{4} \mathrm{X} \mathrm{P}_{6}$ & $-7.53 * *$ & 4.90 & $36.84 * *$ & $5.94 * *$ & $15.93 * *$ \\
\hline $\mathrm{P}_{5} \mathrm{X} \mathrm{P}_{6}$ & $-10.21 * *$ & $-8.78 *$ & 6.76 & $2.85 * *$ & $15.93 * *$ \\
\hline $\mathrm{P}_{6} \mathrm{X} \mathrm{P}_{7}$ & $-11.92 * *$ & -4.90 & $75.54 * *$ & $-12.39 * *$ & $-10.25 * *$ \\
\hline $\mathrm{P}_{6} \mathrm{X} \mathrm{P}_{8}$ & $-6.66 * *$ & -6.20 & 8.68 & $-0.87 *$ & $10.82 * *$ \\
\hline $\mathrm{P}_{6} \mathrm{X} \mathrm{P}_{9}$ & $-10.75 * *$ & $-13.95 * *$ & -6.50 & $6.93 * *$ & $10.15 * *$ \\
\hline $\mathrm{P}_{7} \mathrm{X} \mathrm{P}_{8}$ & $-8.72 * *$ & -2.41 & 12.19 & $-9.91 * *$ & $17.94 * *$ \\
\hline $\mathrm{P}_{7} \mathrm{X} \mathrm{P}_{9}$ & $-17.62 * *$ & $-9.26 *$ & -7.51 & $-13.22 * *$ & $-11.53 * *$ \\
\hline $\mathrm{P}_{9} \mathrm{X} \mathrm{P}_{10}$ & $-13.23 * *$ & -7.59 & $33.11 * *$ & $-4.306 * *$ & $-7.78 * *$ \\
\hline $\mathrm{P}_{11} \mathrm{X} \mathrm{P}_{12}$ & $-5.69 * *$ & -1.30 & 9.13 & $-8.41 * *$ & $-27.93 * *$ \\
\hline $\mathrm{P}_{11} \mathrm{X} \mathrm{P}_{13}$ & $-5.82 * *$ & 0.00 & $34.55 * *$ & $-5.17 * *$ & $-18.63 * *$ \\
\hline $\mathrm{P}_{11} \mathrm{X} \mathrm{P}_{14}$ & $-16.4^{* *}$ & -7.59 & $18.21 *$ & -0.42 & $-10.46 * *$ \\
\hline LSD (0.01) & 4.90 & 10.40 & 22.89 & 1.11 & 1.14 \\
\hline LSD (0.05) & 3.69 & 7.84 & 17.25 & 0.83 & 0.86 \\
\hline
\end{tabular}

* Significant at $5 \%$ level of probability

** Significant at $1 \%$ level of probability 


\section{Fruit weight (g)}

Six $F_{1} S$ out of 19 showed significant negative better parent heterosis and four $F_{1} S$ showed significant positive heterosis for fruit weight (Table 2). The heterosis ranged from -42.84 to 21.21 percent. The highest positive better parent heterosis was observed in $\mathrm{P}_{2} \times \mathrm{P}_{8}$ (21.21\%) followed by $\mathrm{P}_{2} \times \mathrm{P}_{6}$ (18.51). It was clear that higher individual fruit weight in a particular $\mathrm{F}_{1}$ did not show high heterosis because of higher performance by the parental line. The significant negative heterosis was observed in $\mathrm{P}_{11} \times \mathrm{P}_{14}(-42.84 \%)$. Ahmad (2002) also reported heterosis for this trait.

\section{Yield per plant (kg)}

Sixteen cross combinations out of 19 showed significant positive heterosis for yield per plant (Table 2). Better parent heterosis ranged from -21.74 to 54.82 percent. The cross combination $\mathrm{P}_{9} \times \mathrm{P}_{10}$ (54.82 \%) showed maximum heterosis followed by $\mathrm{P}_{3} \times \mathrm{P}_{6}(45.99 \%), \mathrm{P}_{1} \times \mathrm{P}_{8}(43.11 \%), \mathrm{P}_{6} \times \mathrm{P}_{8}(38.86 \%)$ and $\mathrm{P}_{2} \times \mathrm{P}_{6}(36.48 \%)$. The cross $\mathrm{P}_{11} \times \mathrm{P}_{12}$ exhibited highest significant negative better parent heterosis (-21.74 \%). Makesh et al. (2002) also reported heterobeltiosis for this trait.

\section{Fruit length (cm)}

For fruit length, only two cross combinations out of 19 showed significant positive better parent heterosis (Table 2). Heterosis ranged from -17.87 to 3.09 percent. The highest positive heterosis for this trait was observed in $\mathrm{P}_{7} \mathrm{x}_{8}$ (3.09\%) followed by $\mathrm{P}_{9} \times \mathrm{P}_{10}(1.62 \%)$. The highest value of significant negative heterosis was observed in $\mathrm{P}_{3} \times \mathrm{P}_{8}(-17.87 \%)$. Singh et al. (1995) and Ahmad (2002) also reported heterosis for fruit length.

\section{Fruit diameter (cm)}

In the case of fruit diameter, eight crosses exhibited significant positive better parent heterosis, one combination exhibited no heterosis, but the rest of the crosses showed significant negative heterosis (Table 2). Heterosis for this trait ranged from -16.37 to 14.11 percent. The highest value of positive heterotic effect was exhibited by the cross $\mathrm{P}_{3} \times \mathrm{P}_{8}(14.11 \%)$. The highest significant negative better parent heterosis was observed in $\mathrm{P}_{7} \times \mathrm{P}_{9}(-16.37 \%)$. Heterosis for fruit breadth in tomato was also reported by Ahmad (2002) and Chaudhury and Khanna (1972).

\section{Brix percent}

Significant positive heterosis over better parent was observed for brix \% in six crosses, one exhibited zero heterosis and others showed negative heterosis. Percent heterosis ranged from-29.12 to 13.11(Table 2). Highest positive 
heterosis was observed in $\mathrm{P}_{1} \times \mathrm{P}_{6}(13.11 \%)$ and highest significant negative heterosis was obtained in $\mathrm{P}_{11} \times \mathrm{P}_{12}$ (-29.12 \%). Ahmad (2002) and Kurian et al. (2001) also reported heterosis for this trait in tomato.

Table 2. Percent heterosis over better parent in 19 tomato hybrids for yield component characters.

\begin{tabular}{|c|c|c|c|c|c|c|}
\hline Crosses & $\begin{array}{c}\text { Fruits/ } \\
\text { plant }\end{array}$ & $\begin{array}{l}\text { Fruit wt } \\
\text { (g) }\end{array}$ & $\begin{array}{l}\text { Yield/ } \\
\text { plant } \\
\text { (kg) }\end{array}$ & $\begin{array}{l}\text { Fruit } \\
\text { length } \\
(\mathrm{cm})\end{array}$ & $\begin{array}{c}\text { Fruit } \\
\text { diameter } \\
(\mathrm{cm})\end{array}$ & Brix \% \\
\hline $\mathrm{P}_{1} \mathrm{X} \mathrm{P}_{6}$ & $16.76 * *$ & -10.00 & $5.21 * *$ & $-14.26 * *$ & $-5.15 * *$ & $13.11 * *$ \\
\hline $\mathrm{P}_{1} \mathrm{X} \mathrm{P}_{7}$ & 0.17 & -7.80 & $28.76 * *$ & -0.32 & 0.00 & $-13.63 * *$ \\
\hline $\mathrm{P}_{1} \mathrm{X} \mathrm{P}_{8}$ & $49.02 * *$ & -10.00 & $43.11 * *$ & $-6.81 * *$ & $-8.45 * *$ & $2.07 * *$ \\
\hline $\mathrm{P}_{2} \mathrm{X} \mathrm{P}_{6}$ & $18.73 * *$ & $18.51 * *$ & $36.48 * *$ & $-6.97 * *$ & $9.22 * *$ & $4.46 * *$ \\
\hline $\mathrm{P}_{2} \mathrm{X} \mathrm{P}_{7}$ & $16.90 * *$ & $18.18 * *$ & $35.42 * *$ & 0.24 & $6.17 * *$ & $-9.08 * *$ \\
\hline $\mathrm{P}_{2} \mathrm{X} \mathrm{P}_{8}$ & $12.64 * *$ & $21.21^{* *}$ & $25.64 * *$ & $-3.02 * *$ & $1.11 * *$ & $11.78 * *$ \\
\hline $\mathrm{P}_{3} \mathrm{X} \mathrm{P}_{6}$ & $30.71 * *$ & $-14.31 *$ & $45.99 * *$ & $-16.57 * *$ & $8.06 * *$ & $1.14 *$ \\
\hline $\mathrm{P}_{3} \mathrm{X} \mathrm{P}_{8}$ & $10.69 *$ & -1.55 & $26.70 * *$ & $-17.87 * *$ & $14.11 * *$ & $-7.57 * *$ \\
\hline $\mathrm{P}_{4} \mathrm{X} \mathrm{P}_{6}$ & $28.25 * *$ & $-24.31 * *$ & $16.58 * *$ & $-10.36 * *$ & $-9.87 * *$ & $4.00 * *$ \\
\hline $\mathrm{P}_{5} \mathrm{X} \mathrm{P}_{6}$ & $67.44 * *$ & -4.97 & $20.22 * *$ & $-11.91 * *$ & $-4.43 * *$ & 0.69 \\
\hline $\mathrm{P}_{6} \mathrm{X} \mathrm{P}_{7}$ & -8.09 & -2.91 & $-7.83 * *$ & $-17.44 * *$ & $-3.65 * *$ & $-6.48 * *$ \\
\hline $\mathrm{P}_{6} \mathrm{X} \mathrm{P}_{8}$ & $22.3 * *$ & $-15.74 * *$ & $38.86 * *$ & $-3.94 * *$ & $-9.33 * *$ & $-9.73 * *$ \\
\hline $\mathrm{P}_{6} \mathrm{X} \mathrm{P}_{9}$ & -8.61 & -1.25 & $18.44 * *$ & $-13.98 * *$ & $-9.06 * *$ & 0.69 \\
\hline $\mathrm{P}_{7} \mathrm{X} \mathrm{P}_{8}$ & 8.62 & -4.81 & $11.14 * *$ & $3.09 * *$ & $3.27 * *$ & 0.00 \\
\hline $\mathrm{P}_{7} \mathrm{X} \mathrm{P}_{9}$ & $-43.3 * *$ & $-19.48 * *$ & $-20.83 * *$ & $-9.13 * *$ & $-16.37 * *$ & $-20.76 * *$ \\
\hline $\mathrm{P}_{9} \mathrm{X} \mathrm{P}_{10}$ & 4.08 & $11.86 *$ & $54.82 * *$ & $1.62 * *$ & $4.40 * *$ & $-23.60 * *$ \\
\hline $\mathrm{P}_{11} \mathrm{X} \mathrm{P}_{12}$ & $-29.66 * *$ & -4.04 & $-21.74 * *$ & $-6.03 * *$ & $-4.18 * *$ & $-29.12 * *$ \\
\hline $\mathrm{P}_{11} \mathrm{X} \mathrm{P}_{13}$ & $23.56 * *$ & $-15.3 * *$ & $25.15 * *$ & $-3.54 * *$ & $1.82 * *$ & $-10.9 * *$ \\
\hline $\mathrm{P}_{11} \mathrm{X} \mathrm{P}_{14}$ & -5.15 & $-42.84^{* *}$ & $1.53 * *$ & $-14.57 * *$ & $-10.05 * *$ & $-6.67 * *$ \\
\hline $\mathrm{LSD}(0.01)$ & 12.31 & 15.08 & 1.01 & 0.50 & 0.50 & 1.33 \\
\hline $\operatorname{LSD}(0.05)$ & 9.27 & 11.36 & 0.77 & 0.38 & 0.40 & 0.85 \\
\hline
\end{tabular}

* Significant at $5 \%$ level of probability

** Significant at $1 \%$ level of probability

\section{B. Fruit quality attributes}

\section{Fruit shape}

Among the crosses, only two hybrids $\mathrm{P}_{2} \times \mathrm{P}_{6}$ and $\mathrm{P}_{6} \times \mathrm{P}_{9}$ produced slightly flattened, eleven produced round, five produced high round fruits. Only one hybrid produced heart shaped fruits (Table 3). Shape of fruits in tomato was also reported by Morimoto et al. (2000). 


\section{Pedicel area}

Among the nineteen hybrid, only one was flat, the rest were depressed (Table 3). These results also support the findings of Butler (1936).

\section{Shape of pistil scar}

Of the nineteen crosses, six were dot, twelve were stellate and only one was linear shape (Table 3). Khan et al. (1981) reported similar shape.

\section{Blossom end shape}

Among the nineteen crosses, five were indented, fourteen were flat shaped (Table 3). Blossom end shape of fruits in tomato was also reported by Vander Knaap and Tanksley (2003).

Table 3. External fruit characters of $\mathbf{1 9}$ hybrids of tomato.

\begin{tabular}{|c|c|c|c|c|}
\hline Crosses & Fruit shape & Pedicel Area & $\begin{array}{c}\text { Shape of pistil } \\
\text { scar }\end{array}$ & $\begin{array}{c}\text { Blossom end } \\
\text { shape }\end{array}$ \\
\hline $\mathrm{P}_{1} \mathrm{X} \mathrm{P}_{6}$ & Round & Depressed & Stellate & Flat \\
\hline $\mathrm{P}_{1} \mathrm{X} \mathrm{P}_{7}$ & Round & Depressed & Stellate & Flat \\
\hline $\mathrm{P}_{1} \mathrm{X} \mathrm{P}_{8}$ & Round & Depressed & Dot & Flat \\
\hline $\mathrm{P}_{2} \mathrm{X} \mathrm{P}_{6}$ & Slightly flattened & Depressed & Linear & Flat \\
\hline $\mathrm{P}_{2} \mathrm{X} \mathrm{P}_{7}$ & Round & Depressed & Stellate & Flat \\
\hline $\mathrm{P}_{2} \mathrm{X} \mathrm{P}_{8}$ & High round & Depressed & Stellate & Flat \\
\hline $\mathrm{P}_{3} \mathrm{X} \mathrm{P}_{6}$ & Round & Depressed & Stellate & Flat \\
\hline $\mathrm{P}_{3} \mathrm{X} \mathrm{P}_{8}$ & Round & Depressed & Stellate & Flat \\
\hline $\mathrm{P}_{4} \mathrm{X} \mathrm{P}_{6}$ & High round & Depressed & Dot & Flat \\
\hline $\mathrm{P}_{5} \mathrm{X} \mathrm{P}_{6}$ & Round & Depressed & Stellate & Flat \\
\hline $\mathrm{P}_{6} \mathrm{X} \mathrm{P}_{7}$ & Round & Depressed & Dot & Flat \\
\hline $\mathrm{P}_{6} \mathrm{X} \mathrm{P}_{8}$ & High round & Depressed & Dot & Indented \\
\hline $\mathrm{P}_{6} \mathrm{X} \mathrm{P}_{9}$ & Slightly flattened & Depressed & Stellate & Indented \\
\hline $\mathrm{P}_{7} \mathrm{X} \mathrm{P}_{8}$ & High round & Flat & Dot & Flat \\
\hline $\mathrm{P}_{7} \mathrm{X} \mathrm{P}_{9}$ & Round & Depressed & Stellate & Flat \\
\hline $\mathrm{P}_{9} \mathrm{X} \mathrm{P}_{10}$ & Round & Depressed & Stellate & Indented \\
\hline $\mathrm{P}_{11} \mathrm{X} \mathrm{P}_{12}$ & Heart shaped & Depressed & Stellate & Flat \\
\hline $\mathrm{P}_{11} \mathrm{X} \mathrm{P}_{13}$ & High round & Depressed & Dot & Indented \\
\hline $\mathrm{P}_{11} \mathrm{X} \mathrm{P}_{14}$ & Round & Depressed & Stellate & Indented \\
\hline
\end{tabular}


Table 4. Internal fruit characters of 19 hybrids of tomato.

\begin{tabular}{|c|c|c|c|c|}
\hline Crosses & Fruit firmness & Fruit fleshiness & Fruit seediness & No. of locules \\
\hline $\mathrm{P}_{1} \mathrm{X} \mathrm{P}_{6}$ & Good & Good & Medium & 3 \\
\hline $\mathrm{P}_{1} \mathrm{X} \mathrm{P}_{7}$ & Good & Good & Medium & 4 \\
\hline $\mathrm{P}_{1} \mathrm{X} \mathrm{P}_{8}$ & Good & Good & Less & 6 \\
\hline $\mathrm{P}_{2} \mathrm{X} \mathrm{P}_{6}$ & Medium & Good & Less & 4 \\
\hline $\mathrm{P}_{2} \mathrm{XP}_{7}$ & Good & Good & Less & 5 \\
\hline $\mathrm{P}_{2} \mathrm{X} \mathrm{P}_{8}$ & Medium & Medium & Medium & 4 \\
\hline $\mathrm{P}_{3} \mathrm{X} \mathrm{P}_{6}$ & Medium & Medium & Medium & 3 \\
\hline $\mathrm{P}_{3} \mathrm{X} \mathrm{P}_{8}$ & Poor & Poor & Medium & 4 \\
\hline $\mathrm{P}_{4} \mathrm{X} \mathrm{P}_{6}$ & Good & Good & Less & 3 \\
\hline $\mathrm{P}_{5} \mathrm{X} \mathrm{P}_{6}$ & Medium & Medium & Medium & 5 \\
\hline $\mathrm{P}_{6} \mathrm{X} \mathrm{P}_{7}$ & Medium & Good & Less & 7 \\
\hline $\mathrm{P}_{6} \mathrm{X} \mathrm{P}_{8}$ & Good & Good & Less & 3 \\
\hline $\mathrm{P}_{6} \mathrm{X} \mathrm{P}_{9}$ & Good & Medium & Highly & 6 \\
\hline $\mathrm{P}_{7} \mathrm{X} \mathrm{P}_{8}$ & Good & Good & Medium & 4 \\
\hline $\mathrm{P}_{7} \mathrm{X} \mathrm{P}_{9}$ & Medium & Poor & Highly & 6 \\
\hline $\mathrm{P}_{9} \mathrm{X} \mathrm{P}_{10}$ & Poor & Poor & Highly & 3 \\
\hline $\mathrm{P}_{11} \mathrm{X} \mathrm{P}_{12}$ & Good & Medium & Medium & 4 \\
\hline $\mathrm{P}_{11} \mathrm{X} \mathrm{P}_{13}$ & Good & Medium & Medium & 3 \\
\hline $\mathrm{P}_{11} \mathrm{X} \mathrm{P}_{14}$ & Good & Good & Less & 3 \\
\hline
\end{tabular}

\section{Fruit firmness}

Fruit firmness of eleven hybrids were found good, six were medium and two were poor (Table 4). These results also support the findings of Harker et al. (1997).

\section{Fruit fleshiness}

Fruit fleshiness of ten hybrids was good, six had medium and three had poor (Table 4). We observed that fruit fleshiness of most of the hybrids was better. Fruit fleshiness in tomato was also reported by Harker et al. (1997).

\section{Fruit seediness}

Among the crosses, seven were less seeded, nine were medium and three were high seeded (Table 4). These results also support the findings of Sako et al. (2001). 


\section{Locules per fruit}

Among all the crosses, seven had three locules, six had four lucules, two had five, three had six and one had seven locules (Table 4). Kurian et al. (2001) also reported heterosis number of loculer per fruit.

Considering all the parameters, the cross combinations $\mathrm{P}_{1} \times \mathrm{P}_{8}, \mathrm{P}_{2} \times \mathrm{P}_{6}, \mathrm{P}_{2} \mathrm{x}$ $\mathrm{P}_{7}, \mathrm{P}_{2} \times \mathrm{P}_{8}, \mathrm{P}_{3} \times \mathrm{P}_{8}, \mathrm{P}_{5} \times \mathrm{P}_{6}$ showed high heterotic performance with better desirable characters, so recommended for further studies as new varieties of commercial cultivation.

\section{References}

Ahmad, S. 2002. Genetics of fruit set and related traits in tomato under hot-humid conditions. Ph. D. Thesis. Bangabandhu Sheikh Mujibur Rahman Agricultural University. Salna, Gazipur. pp. 1-236.

Anonymous. 1995. Agro-climotological data. Agromet Division, Bangladesh Metrological Department, Joydebpur, Gazipur. pp. 35-65.

BBS. 2007. Statistical Year Book of Bangladesh, Bangladesh Bureau of Statistics, Ministry of Planning, Govt. of Bangladesh. p.139.

Butler, L. 1936. Inherited characters in the tomato. II. Jointless pedicel. J. Hered. 37: 25-26.

Chaudhury, R. C. and K. R. Khanna. 1972. Exploitation of heterosis in tomato yield and components. South Indian Hort. 20: 59-65.

Gomez, K. A. and Gomez, A. A. 1984. Statistical procedures for Agricultural Research. John Willey and Sons. Inc. New York. pp. 67-215.

Harker, F. R.; R. J. Redgwell; I. C. Hallett; S. H. Murray. 1997. Texture of Fresh Fruit. Hortic. Rev. 20: 121-224.

Khan, M. R. I., S. Khanum, M. A. Q. Shaikh and A. K. Kaul.1981. Genetic studies in tomato (Lycopersicon esculentum). Some qualitative characters. Bangladesh J. Bot. 10(2): 157-263.

Kurian, A.; K. V. Petter and S. Rajan. 2001. Heterosis for yield components and fruit characters in tomato. J. Tropical Agric. 39(1): 5-8.

Makesh, S.; M. Puddan; S. Ashok and M. R. Banu. 2002. Heterosis studies for quality and yield in tomato (Lycopersicon esculentum Mill.). Advances in Plant Science 15(2):597-601.

Morimoto T; T. Takeuchi; H. Miyata; Y. Hashimoto. 2000. Pattern recognition of fruit shape based on the concept of chaos and neural networks. Comp Electron Agric. 26: 171-186.

Razzaque, M. A., M. A. Satter, M. A. Quayyum and M. S. Alam. 2000. Krishi Projukti Hatboi (Hand book of Agro-technology) $2^{\text {nd }}$ edn, Bangladesh Agricultural Research Institute, Gazipur 1701: pp. 1-464. 
Resende L. V., W. R. Maluf, J. T. V. Resende, L. A. A. Gomes. 2000. Combining ability of oblong-fruit tomato breeding lines with different genetic controls and levels of tospovirus resistance. Ciencia-e-Agrotechnologia 24(3):549-559. (in Portuguese)

Sako Y; M. B. McDonald; K. Fujimura; A. F. Evans; M. A. Bennett. 2001. A system for automated seed vigour assessment. Seed Sci Technol 29: 625-636.

Singh, A.; P. K. Singh; J. Dixit and J. P. S. Guatam. 1995. Heterosis and inbreeding depression in tomato. Hort. J. 8(2): 125-129.

Tesi, R.; A. Grainfenberg and M. E. Creatim.1970. Heterosis and quality in $F_{1}$ hybrids of L esculentum. Mill., growth under glass. Riv. Ortoflorofruttic Ital. 54: 269-292.

Vander Knaap E; S. D. Tanksley. 2003. The making of a bell pepper-shaped tomato fruit: identification of loci controlling fruit morphology in Yellow Stuffer tomato. Theor Appl Genet. 107: 139-147. 